\title{
Genetic Analysis of Anthropometric Measures in 11-Year-Old Twins: The Medical College of Virginia Twin Study
}

\author{
JOANN N. BODURTHA, MICHAEL MOSTELLER, JOHN K. HEWITT, WALTER E. NANCE, \\ LINDON J. EAVES, WILLIAM B. MOSKOWITZ, SOLOMON KATZ, AND \\ RICHARD M. SCHIEKEN
}

The Children's Medical Center. Division of Pediatric Cardiology and Department of Human Genetics, Medical College of Virginia. Virginia Commonwealh University, Richmond, Virginia and The Krogman Growth Center at The University of Pennsylvania, Philadelphia, Pennsylvania

\begin{abstract}
We have conducted a cross-sectional analysis of the genetic and environmental contributions to the variance of anthropometric measurements in children during early adolescence. Univariate path analysis was used to estimate the relative contributions of genes, individual environment, and family environment to measures of childhood obesity in 259 11-y-old Caucasian twin pairs. Triceps, subscapular, and suprailiac skinfold thicknesses, as well as waist circumferences, ht, and wt were measured in a standardized protocol. In this sample, a parsimonious model that included only additive genetic effects and environmental factors unique to the individual provided an adequate explanation for the variation in ht, wt, quetelet index, and subscapular and triceps skinfolds. In this largely preadolescent population, different magnitudes of genetic effects were seen in males and females for waist circumference, biiliac diameter, and suprailiac skinfold. (Pediatr Res 28: 1-4, 1990)
\end{abstract}

\section{Abbreviations}

MZ, monozygotic

$\mathrm{DZ}$, dizygotic

Childhood body habitus, especially obesity, is influenced by a variety of factors and may have an important relationship to the later development of heart disease. In adults, obesity may act as an independent risk factor for coronary heart disease or contribute to the morbidity of other risk factors and predispositions ( 1 , 2). To explore the possible childhood antecedents of obesity, we have investigated several measures of adiposity and body habitus in a large sample of 11-y-old twins. Previous twin and family studies of these anthropometric measures have generally been based on subjects that were heterogenous with respect to age and have excluded unlike-sexed pairs, thus losing the information these subjects can provide about the homogeneity of any obscured genetic or environmental effects across the sexes. In a large sample of prepubertal Caucasian twins of known sexual maturity stages and uniform age, we investigated the underlying genetic and environmental variation of standardized measurements of adiposity at several body sites.

Received May 17, 1989; accepted March 13,1990.

Correspondence and reprint requests: Richard M. Schieken, M.D., Box 26 MCV Station. Medical College of Virginia, Richmond. VA 23298-0026.

Supported by the National Institules of Health, National Heart, Lung and Blood Institute (R0) HL31010). This is paper 316 from the Department of Human Genetics.

\section{MATERIALS AND METHODS}

As part of an ongoing longitudinal study of developmental changes and the genetic determinants of blood pressure during adolescence, we ascertained from school rosters 259 Caucasian twin pairs living together in Virginia. The sample included 73 $\mathrm{MZ}$ male pairs, $75 \mathrm{MZ}$ female pairs, 25 like-sex male $\mathrm{DZ}$ pairs, 31 like-sex female $\mathrm{DZ}$ pairs, and 54 unlike-sex $\mathrm{DZ}$ pairs who were examined as near to their 1lth birthdays as possible. All twins were reared together. Informed consent was obtained from the twins and their parents for participation in an institutionally approved research protocol. Zygosity was determined as previously described (3) with a battery of blood group, enzyme, and HLA testing. Skinfold thicknesses of triceps, subscapular, and supratliac areas were measured by the technique of Tanner (4) with Holtain skinfold calipers designed to exert a constant pressure of $10 \mathrm{~g} / \mathrm{mm}^{2}$ over a range of $0-48 \mathrm{~mm}$. Sexual maturity stages were assessed by the scales of Marshall and Tanner $(5,6)$. Waist circumferences were measured at the narrowest part of the trunk and biliac diameters at the iliac crest. Wt and ht were measured by trained examiners using a conventional balanced scale and stadiometer. Quetelet index was calculated as wt $/(\mathrm{ht})^{2}$.

Statistical analysis. Much of the preliminary statistical work was carried out using the SAS (Cary, NC) computer package. Male-female mean differences were assessed by a pooled $t$ test analysis, which took into account whether or not the two groups had different variances. The correlations used to summarize the twin-twin covariation by zygosity and sex type are Pearson product-moment correlations. Within like-sex twin pairs, the first-born twin was consistently designated as twin 1 , and the cotwin as twin 2 . In the unlike-sex twin pairs, the male co-twin was designated as twin 1 and the female as twin 2 . To pool correlation coefficients within a zygosity group across sex types and to test for homogeneity among the coefficients, a z-score procedure outlined by Steele and Torrie (7) was used. Because the parameter estimation techniques used assume that variables are normally distributed, all of the variables that exhibited significant skewing were adjusted to normality by a logarithmic transformation before the analysis. A variety of causal models were hypothesized, by means of path diagrams (8), to explain the observed variances and covariances of the research measures. Path analysis is used to explain variation in a measured variable. The variation is caused by a combination of genetic and environmental effects. We can further partition the genetic effects into those specific for boys and girls and the environmental effects into those specific for a twin and shared by a twin.

A path diagram is a graphic depiction of a causal model that is postulated to explain the observed variation and covariation among sets of measured dependent variables. Thus in Figures 1 


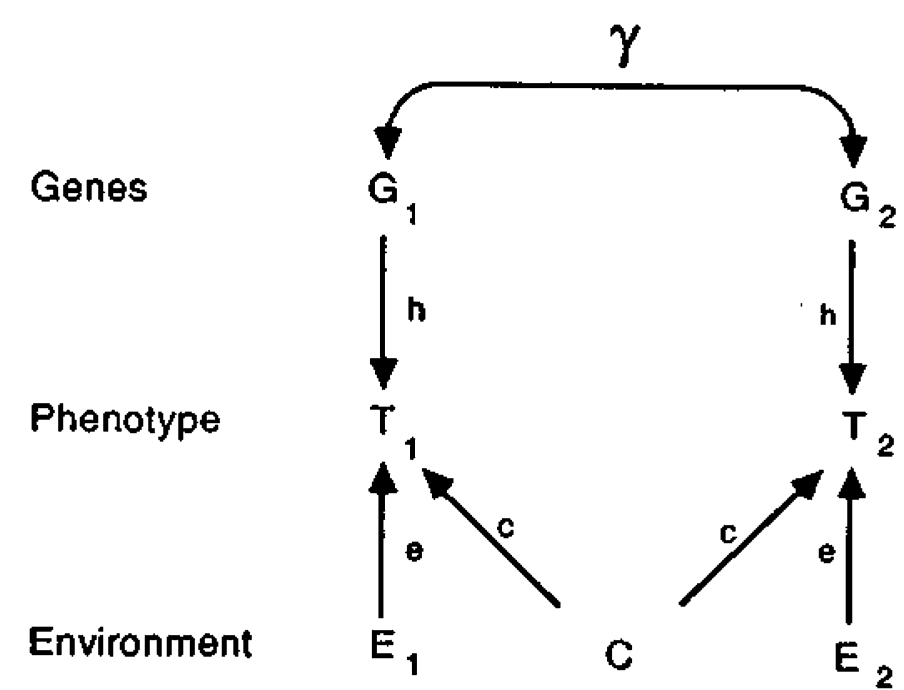

Fig. 1. Path diagram for like-sex twin pairs. The phenorypes of the twins $\left(T_{1}, T_{2}\right)$ are modeled as being determined by additive genetic effects $\left(G_{1}, G_{2}\right)$, environmental effects common to both twins $(C)$, and environmental effects specific to each twin $\left(E_{1}, E_{2}\right)$. $\gamma$ is the correlation of genetic effects on twin 1 with those on twin 2. For MZ twins, $\gamma=1$. For $\mathrm{DZ}$ twins, $\gamma=0.5$.

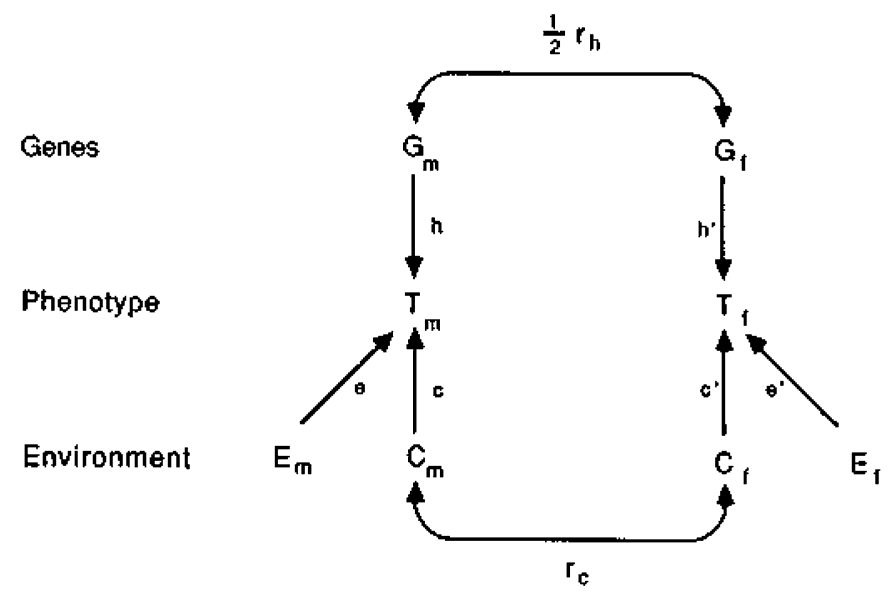

Fig. 2. Path diagram for unlike-sex (DZ) twin pairs. Additive genetic, common environmental, and specific environmental effects are allowed to exert different effects upon the male twin and female twin. $\digamma_{h}$ represents the correlation between the genetic effects in the male and female twins. $r_{c}$ represents the correlation of the effects of the common (shared) environment on the male and female twins.

and 2 , the phenotypic covariation between the observed anthropometric measurements of twins (i.e. $T_{1}$ and $T_{2}$ ) is assumed to arise either from the unobserved correlated effects of the genes $\left(G_{1}\right.$ and $\left.G_{2}\right)$ they share or from common or shared influences of the home environment (C). Whereas the intrauterine environments of $\mathrm{MZ}$ and $\mathrm{DZ}$ twins may differ, the effects of these biases would be to reduce the observed correlations of MZ twins in comparison with $\mathrm{DZ}$ pairs thus rendering the resulting estimates of genetic variance somewhat conservative. $C$ is assumed to be the same for both identical and fraternal twins. Random environmental factors, including measurement error, $\left(E_{1}\right.$ and $\left.E_{2}\right)$, that are unique to individual twins also contribute to the phenotypic variance but are uncorrelated as designated by the absence of a connecting arc in the diagram. The correlation between the genotypes of identical or monozygotic twins is 1 , whereas DZ pairs, being no more alike genetically than full siblings, share only half of their genes and have a genetic correlation of 0.5 . The magnitude of the coefficients for the paths connecting the unobserved (e.g. G, E, etc.) and observed variables provide a measure of the strength or relative intensity of the postulated latent causes. For example, the closer in magnitude MZ and DZ twin correlations are, the larger the contribution common environment would be expected to make.

Unlike-sex DZ pairs are frequently omitted from twin studies in the mistaken belief that their exclusion will "control" for sex differences. In reality, the inclusion of unlike-sex pairs in an overall analysis provides an opportunify to test for the possibility that the set of genes that influences a particular variable in males may be different from the set of genes that influences the variable in females.

The LISREL VI program (9) was used to obtain maximum likelihood estimates of model parameters and a $\chi^{2}$ goodness-offit statistic. Comparisons of alternative models with equal numbers of parameters were based on this goodness of fit statistic, the model with the lower value being the preferred one. Models with different numbers of parameters were compared by subtracting their $x^{2}$ values and their $d f$ and observing whether the inclusion of addition parameters resulting in a statistically significant decrease in the goodness-of-fit statistic. For example, if the $\chi^{2}$ for a model including $h, c$, and $\mathrm{e}$ (genetic effects, shared and individual environmental effects, respectively) were 6.1 and the model that included only $h$ and $e$ had $\chi^{2}$ of 6.8 , then the difference $\chi^{2}$ would be 0.7 and would be associated with a probability of about $0.40\left(x^{2}, 1 d f\right)$. We would conclude that the inclusion of the parameter $c$ did not result in a better fit and therefore the simpler of the two models, the h-e model, would be preferred.

Two aspects of male-female genetic control differences were explored using the model fitting approach. The first deals with the question: "Are the magnitudes of the effects equal in males and females?" To address this question, one model was fit that allowed the magnitudes of the effects to be different for males and females and another was fit that constrained the magnitudes to be equal. If the former model provided a statistically better fit, this was taken as evidence for a difference in magnitude of the effects in males and females. Another question regarding genetic control is whether the genes that influence a particular trait in males are the same genes that influence the trait in females. If completely different sets of genes influence a trait in males and females, a reduction in the correlation between unlikesex twins, relative to like-sex $D Z$ twins, would be expected. The parameter in the model that reflects this is $\Gamma_{h}$. When this parameter is estimated to be 1 , it implies that the same genes control the trait in males and females, whereas, when $\boldsymbol{r}_{\mathrm{h}}$ is 0 , it implies that completely different sets of genes are active in males and females.

The simplest models that fit the observed statistics and the parameter estimates, as a percent of the total variance, are given below.

\section{RESULTS}

The mean age of the twins was $11.1 \pm 0.3 \mathrm{y}$ with a range of 10.9 to $11.9 \mathrm{y}$. The means and SD for the untransformed size and adiposity measures are given for the twin pairs in Table 1. Girls had significantly larger skinfold and biiliac diameter measurements than boys $(p<0.01)$. Boys had larger waist circumferences. Girls were also of somewhat more advanced Tanner sexual maturity stage $(2.19 \pm 0.94$ versus $1.72 \pm 0.63$ with a range of $1-5$ for girts and $1-4$ for boys).

Pearson correlation coefficients for the transformed variables by twin type (twin 1 versus twin 2) are shown in Table 2. For all measurements, the MZ twins were highly correlated, whereas lower correlations were seen in the $\mathrm{DZ}$ twins.

The model-fitting results are summarized in Table 3. Maximum likelihood parameter estimates are presented for the most parsimonious model that fits the data. Parameters are expressed as estimated percentages of the total phenotypic variance due to the three main sources: genes $\left(h^{2}\right)$, shared environment $\left(c^{2}\right)$, and 